\title{
Clinical outcomes of nonelective coronary revascularization with and without cardiopulmonary bypass
}

Sotiris C. Stamou, MD, PhD, ${ }^{a}$ Peter C. Hill, MD, ${ }^{b}$ Elizabeth Haile, MS, ${ }^{c}$ Syma Prince, RN, ${ }^{d}$ Michael J. Mack, MD, ${ }^{d}$ and Paul J. Corso, MD

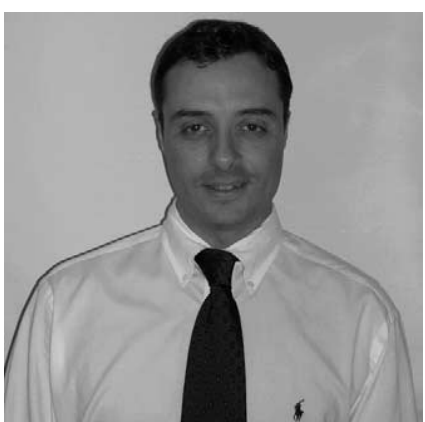

Dr Stamou
From the Department of Cardiac Surgery, ${ }^{\text {a }}$ Cleveland Clinic Foundation, Cleveland, Ohio; the Section of Cardiac Surgery, ${ }^{\text {b }}$ Washington Hospital Center, Washington, DC; the MedStar Research Institute, ${ }^{\mathrm{c}}$ Washington, DC; and the Cardiovascular Research Science and Technology Institute, ${ }^{\mathrm{d}}$ Dallas, Tex.

Received for publication May 25, 2005; revisions received Aug 26, 2005; accepted for publication Aug 31, 2005.

Accepted for publication Aug 31, 2005.

Address for reprints: Paul J. Corso, MD, Section of Cardiac Surgery, Washington Hospital Center, 106 Irving Street NW, Suite 316, South Tower, Washington, DC 20010 (E-mail: paul.j.corso@ MedStar.net).

J Thorac Cardiovasc Surg 2006;131:28-33

$0022-5223 / \$ 32.00$

Copyright (C) 2006 by The American Association for Thoracic Surgery

doi:10.1016/j.jtcvs.2005.08.059
Background: Patients who undergo emergent coronary artery bypass grafting pose a greater challenge in terms of intraoperative and postoperative mortality and morbidity compared to elective coronary artery bypass. Coronary artery bypass without cardiopulmonary bypass (off-pump coronary artery bypass) might benefit these high-risk patients by eliminating the cardiopulmonary bypass and therefore decreasing the systemic inflammatory response associated with it. The aim of this study was to compare the early clinical outcome after nonelective off-pump coronary artery bypass with a matched set of patients undergoing coronary artery bypass with cardiopulmonary bypass (on pump).

Methods: Between January 2000 and October 2003, 2273 patients underwent nonelective (urgent or emergent) off-pump coronary artery bypass and were compared with a contemporaneous control group of 3487 patients undergoing on-pump coronary artery bypass. Logistic regression analysis was used to compare operative mortality, postoperative stroke, length of stay, postoperative placement of intra-aortic balloon pump, postoperative renal failure, and hemorrhage-related re-exploration between the groups, controlling for preoperative risk factors. The patients undergoing off-pump coronary artery bypass were matched to patients undergoing on-pump bypass by propensity scores.

Results: Patients undergoing off-pump coronary artery bypass had comparable operative mortality (odds ratio, 0.8 ; 95\% confidence interval, $0.57-1.15 ; P=.24$ ) and stroke rate (odds ratio, 0.6 ; 95\% confidence interval, $0.33-1.08 ; P=.09$ ) with the patients undergoing on-pump coronary artery bypass after controlling for preoperative risk factors through matching. Off-pump coronary artery bypass was associated with an abbreviated length of stay (odds ratio, $0.5 ; 95 \%$ confidence interval, $0.47-0.64 ; P<.01$ ), lower rate of postoperative renal failure (odds ratio, $0.5 ; 95 \%$ confidence interval, $0.37-0.72 ; P<.01$ ), intra-aortic balloon pump placement (odds ratio, 0.5 ; 95\% confidence interval, $0.3-0.71 ; P<.01$ ), and hemorrhage-related re-exploration rate (odds ratio, $0.70 ; 95 \%$ confidence interval, $0.5-1.0 ; P=.5$ ) compared with on-pump coronary artery bypass after matching by propensity scores.

Conclusions: Nonelective coronary revascularization without cardiopulmonary bypass is associated with comparable operative mortality and stroke and abbreviated length of stay. Off-pump coronary artery bypass might also decrease the need for intra-aortic balloon pump placement and lower the rate of postoperative renal failure and hemorrhage-related re-exploration compared with that of conventional on-pump coronary artery bypass in this subset of patients.

I ndications for CABG are now well defined, with an apparent shift to more advanced coronary disease and extensive comorbidity. ${ }^{1}$ Several studies have reported that in spite of an increasingly high-risk population, CABG continues to show favorable short- and long-term outcomes. ${ }^{2}$ Nevertheless, it is well recognized that despite the improvement in surgical techniques, postoperative care, and anesthesia and a better understanding of the physiology of high-risk patients, CABG 

Abbreviations and Acronyms
$\mathrm{BMI}=$ body mass index
$\mathrm{CABG}=$ coronary artery bypass grafting
$\mathrm{CI}=$ confidence interval
IABP $=$ intra-aortic balloon pump
OR = odds ratio

with cardiopulmonary bypass (on-pump CABG) is associated with substantial morbidity, which is ascribed to the adverse effects of extracorporeal circulation. ${ }^{3,4}$

CABG without cardiopulmonary bypass (off-pump CABG) has been explored as an emerging technique for coronary revascularization by circumventing the adverse effects of cardiopulmonary bypass. ${ }^{5-7}$ Over the past 5 years, the number of procedures performed with off-pump CABG has increased significantly. Previous studies showed a decrease in risk-adjusted complication rates and in 30-day mortality rates when comparing off-pump $\mathrm{CABG}$ with the conventional on-pump CABG procedure. ${ }^{5-8}$

However, there are few reports and comparative studies on outcomes with off-pump CABG in urgent and emergent cases versus the conventional approach with cardiopulmonary bypass. ${ }^{9,10}$ Patients who undergo emergent or urgent CABG pose a greater challenge in terms of intraoperative and postoperative mortality and morbidity. Off-pump CABG might benefit these high-risk patients by eliminating the cardiopulmonary bypass and therefore decreasing the systemic inflammatory response associated with it. Preliminary reports have revealed improved outcomes associated with nonelective off-pump CABG in terms of mortality and morbidity ${ }^{9,10}$ compared with conventional on-pump CABG. These reports, however, are limited by small sample size, inappropriate statistical methodology, or both.

The aim of this study was to compare the early clinical outcome after nonelective off-pump CABG with that in a matched set of patients undergoing on-pump CABG.

\section{Patients and Methods}

\section{Patients}

The computerized database of the Section of Cardiac Surgery of the Washington Hospital Center, Washington, DC, and Cardiovascular Research Science and Technology Institute at Dallas, Texas, was queried to identify all patients who underwent nonelective CABG between January 2000 and October 2003 ( $n=6260)$. Patients who underwent minimally invasive direct coronary artery bypass (through an anterorolateral or posterolateral thoracotomy or through a xiphoid approach) and patients with more than 6 grafts were excluded from this study. The patients excluded from the study $(n=500)$ because of the above exclusion criteria or because of missing essential data were evenly distributed between the 2 groups. Twelve surgeons were contributing cases for the period of study from both institutions. During the period of study, $22.8 \%$ of patients had urgent CABG, $4.66 \%$ of patients had emergent $\mathrm{CABG}, 0.34 \%$ had salvage $\mathrm{CABG}$, and $72 \%$ of patients had elective $\mathrm{CABG}$. Baseline demographics, procedural data, and perioperative outcomes were recorded and entered into a prespecified database by a dedicated data-coordinating center. In general, patients undergoing urgent $\mathrm{CABG}$ were considered those who have not been scheduled for routine admission from the waiting list but who require surgical intervention on the same admission for medical reasons. Patients cannot be discharged home without an operation. Patients undergoing emergent $\mathrm{CABG}$ are those with ongoing cardiac compromise in whom there should not be a delay of surgical intervention. Data were analyzed according to the Society of Thoracic Surgeons National Cardiac Surgery Database guidelines and definitions (http://www.ctsnet.org/file/rptDataSpecifications252_1_ ForVendorsPGS.pdf). The classification of each case was performed by the operating surgeon on the basis of the Society of Thoracic Surgeons' definitions.

\section{Sample Size Calculation}

The mortality rates were based on the literature, with rates among the on-pump CABG group being $3.12 \%$ and those among the off-pump CABG group being $1.82 \%$. The publication that the rates are based on is by Magee and colleagues. ${ }^{6}$ Univariate comparison of complications and mortality for the combined institutions for on-pump CABG versus off-pump CABG shows a significantly higher mortality in the on-pump CABG group $(3.12 \%$ vs $1.82 \%$, $P=.002){ }^{6}$ From the calculations above, it is evident that a total of 5740 patients (2870 patients per group) are needed to obtain a study power of $0.8(80 \%)$ with an $\alpha$ value of.017.

The stroke rates were based on the literature, with rates among the on-pump CABG group being 0.024 (2.4\%) and those among the study group being $0.007(0.7 \%)$. The publication that the rates are based on is by Karthik and associates. ${ }^{11}$ Compared with on-pump CABG, off-pump CABG was associated with lower rates of stroke $(0.7 \%$ vs $2.4 \%, P<.047)$. On the basis of these rates, a total of 2200 patients (1100 patients per group) are needed to obtain a study power of $0.8(80 \%)$ with an $\alpha$ value of .017 .

\section{Data Analysis}

Univariate comparisons of preoperative risk factors were performed for patients undergoing nonelective operations between the on-pump and off-pump CABG groups. The preoperative risk factors to be compared were age, sex, number of grafts, diabetes, hypertension, congestive heart failure, hemodialysis, recent myocardial infarction, cerebrovascular accident, renal failure, chronic obstructive pulmonary disease, ejection fraction, reoperative CABG, the Parsonnet Bedside Risk Score, and the Northern New England Stroke Risk Score. Dichotomous variables were compared by using a $\chi^{2}$ test of general association. Comparisons between continuous variables were performed by using the Student $t$ test for normally distributed data or the Wilcoxon rank sum test for nonparametric data. Ordinal data were compared by using the Cochran-Armitage trend test.

Separate logistic regression models were used to determine independent predictors of 30-day (operative) mortality, stroke, intra-aortic balloon pump (IABP) placement, postoperative renal failure, and hemorrhage-related re-exploration. The logistic model 
TABLE 1. Univariate comparisons of preoperative patient characteristics between off-pump and on-pump CABG

\begin{tabular}{|c|c|c|c|}
\hline & On-pump CABG ( $n=3487)$ & Off-pump CABG $(\mathrm{n}=2773)$ & $P$ value \\
\hline Age (y) & $63.1 \pm 10.8$ & $65.1 \pm 11.3$ & $<.01^{*}$ \\
\hline Female sex (vs male sex) & $941(27.0)$ & $946(34.1)$ & $<.01$ \\
\hline Body mass index & $28.0(25.2-31.7)$ & $27.8(24.7-31.3)$ & $<.01 \dagger$ \\
\hline Diabetes & $1115(32.0)$ & $908(32.7)$ & .52 \\
\hline Chronic renal insufficiency & $110(3.2)$ & $149(5.4)$ & $<.01$ \\
\hline Hemodialysis & $19(0.5)$ & $35(1.3)$ & $<.01$ \\
\hline Left main stenosis & $1006(28.9)$ & $605(21.8)$ & $<.01$ \\
\hline Hypertension & $2490(71.4)$ & $1982(71.5)$ & .95 \\
\hline Recent acute myocardial infarction $(<24 \mathrm{~h})$ & $142(4.1)$ & $48(1.7)$ & $<.01$ \\
\hline History of acute myocardial infarction & $1803(51.7)$ & $1327(47.9)$ & $<.01$ \\
\hline Congestive heart failure & $482(13.8)$ & $395(14.2)$ & .63 \\
\hline Urgent case (vs emergency case) & $3170(90.9)$ & $2628(94.8)$ & $<.01$ \\
\hline Carotid artery stenosis & $80(2.3)$ & $72(2.6)$ & .44 \\
\hline Reoperative CABG & $226(6.5)$ & $172(6.2)$ & .65 \\
\hline History of cerebrovascular accident & $152(4.4)$ & $114(4.1)$ & .63 \\
\hline Chronic obstructive pulmonary disease & $61(1.8)$ & $45(1.6)$ & .70 \\
\hline Preoperative intra-aortic balloon pump & $473(13.6)$ & $174(6.3)$ & $<.01$ \\
\hline Peripheral vascular disease & $428(12.3)$ & $357(12.9)$ & .48 \\
\hline Left ventricular ejection fraction & & & $<.01 \ddagger$ \\
\hline$>45 \%$ & $2119(60.8)$ & $1483(53.5)$ & \\
\hline $35 \%-45 \%$ & $728(20.9)$ & $669(24.1)$ & \\
\hline $25 \%-35 \%$ & $443(12.7)$ & $439(15.8)$ & \\
\hline$<25 \%$ & $197(5.7)$ & $182(6.6)$ & \\
\hline No. of grafts & & & $<.01 \ddagger$ \\
\hline 1 & $61(1.8)$ & $254(9.2)$ & \\
\hline 2 & 461 (13.2) & $577(20.8)$ & \\
\hline 3 & $1234(35.4)$ & $921(33.2)$ & \\
\hline 4 & $1178(33.8)$ & 757 (27.3) & \\
\hline 5 & 465 (13.3) & $235(8.5)$ & \\
\hline 6 & $88(2.5)$ & $29(1.1)$ & \\
\hline
\end{tabular}

Values are expressed as $\mathrm{n}(\%)$, mean \pm standard deviation, or median (25th-75th percentile). $\chi^{2}$ Tests are used for all comparisons unless noted otherwise. No adjustments were made for repeated testing. CABG, Coronary artery bypass grafting. *Student $t$ test. $\dagger$ Wilcoxon rank sum test. $\ddagger$ Cochran-Armitage trend test.

consisted of all preoperative risk factors that were deemed significant $(P<.15)$ from the univariate analysis comparing on-pump versus off-pump CABG, risk factors associated with nonelective CABG from the literature, and risk factors from the literature associated with the specific outcome (operative mortality or stroke). Model fit analysis was evaluated by using the HosmerLemeshow goodness-of-fit statistical and residual analysis. The c-statistic is reported as a measure of predictive power. The presence of linear dependencies or correlation among the independent variables was checked by using diagnostics from ordinary logistic regression.

Analysis of covariance was used to compare the means of postoperative length of stay between the 2 groups while adjusting for some risk factors. All the preoperative risk factors that were deemed significant $(P<.15)$ from the univariate analysis of on-pump versus off-pump CABG, risk factors associated with urgent-emergent $\mathrm{CABG}$ from the literature, and risk factors from the literature associated with postoperative length of stay were adjusted for in the analysis of covariance model.

Propensity scores were then computed for all patients and used to match patients from the off-pump and on-pump CABG groups, as previously described. ${ }^{12}$ By matching on the basis of propensity score, a measure of the probability of being selected for either off-pump or on-pump CABG, each group will have nearly equal proportions of the preoperative variables. ${ }^{12,13}$ Matching also controls for confounding variables. The general estimating method was then used in a logistic regression to test the difference in the rate of transfusion and hemorrhage-related re-exploration between the off-pump and on-pump CABG groups in the propensity scorematched subpopulation.

\section{Results}

\section{Unmatched Sample}

Preoperative characteristics. Of the patients who underwent nonelective CABG at the Washington Hospital Center, $55.1 \%$ of operations were performed off pump, whereas of those who underwent CABG at the Cardiovascular Research Science and Technology Institute, 38.6\% of operations were performed off pump. Patient preoperative characteristics are presented in Table 1. Patients who had off-pump CABG were more likely to be female and 
TABLE 2. Univariate comparisons of postoperative patient characteristics between off-pump and on-pump CABG

\begin{tabular}{lccc}
\hline & $\begin{array}{c}\text { On pump } \\
\text { (n = 3487) }\end{array}$ & $\begin{array}{c}\text { Off pump } \\
\text { (n = 2773) }\end{array}$ & P value \\
\hline Stroke & $54(1.6)$ & $36(1.3)$ & .41 \\
Acute myocardial infarction & $54(1.6)$ & $31(1.1)$ & .14 \\
Acute renal failure & $184(5.3)$ & $84(3.0)$ & $<.01$ \\
Intra-aortic balloon pump & $130(3.7)$ & $55(2.0)$ & $<.01$ \\
New-onset atrial fibrillation & $735(21.1)$ & $489(17.6)$ & $<.01$ \\
Hemorrhage-related re- & $132(3.8)$ & $72(2.6)$ & .01 \\
$\quad$ exploration & & & \\
Postoperative length of stay & $6(5-8)$ & $5(4-7)$ & $<.01^{*}$ \\
Operative mortality & $146(4.2)$ & $96(3.5)$ & .14 \\
\hline
\end{tabular}

Values are expressed as $\mathrm{n}(\%)$ or median (25th-75th percentile). All comparisons are made with a $\chi^{2}$ test for independence unless noted otherwise. No adjustments were made for repeated testing. Comparisons of outcomes are unadjusted for risk factors. *Wilcoxon rank sum test.

elderly and more likely to have chronic renal insufficiency and an ejection fraction of less than $35 \%$. Patients who underwent on-pump CABG were more likely to have higher body mass index (BMI), diabetes, left main stenosis, recent myocardial infarction, preoperative IABP placement, and multivessel coronary artery disease.

Postoperative characteristics. Postoperative characteristics are presented in Table 2. In univariate analysis patients who underwent off-pump CABG had a lower incidence of postoperative acute renal failure $(P<.01)$, new-onset atrial fibrillation $(P<.01)$, postoperative IABP placement, hemorrhage-related re-exploration $(P<.01)$, and postoperative length of stay. Operative mortality, stroke, and acute myocardial infarction were comparable between the 2 groups.

Multivariate Analysis. OPERATIVE MORTALITY. Independent predictors of operative mortality identified from the multiple logistic regression model on the unmatched sample included age (odds ratio [OR], 1.05; 95\% confidence interval $[\mathrm{CI}], 1.03-1.06 ; P<.01)$, female sex (OR, $1.9 ; 95 \% \mathrm{CI}$, $1.42-2.50 ; P<.01$ ), chronic renal insufficiency (OR, 2.0; 95\% CI, 1.27-3.29; $P<.01$ ), left main stenosis (OR, 1.5; 95\% CI, 1.12-2.01; $P<.01$ ), history of acute myocardial infarction (OR, 1.7; 95\% CI, 1.22-2.26; $P<.01)$, preoperative IABP placement (OR, 1.8; 95\% CI, 1.21-2.56; $P<.01$ ), ejection fraction of less than $35 \%$ (OR, 2.3 ; $95 \%$ CI, 1.68 3.13; $P<.01$ ), and reoperative $\mathrm{CABG}(\mathrm{OR}, 2.8 ; 95 \% \mathrm{CI}$, $1.86-4.18 ; P<.01)$. Off-pump CABG did not emerge as an independent predictor of operative mortality (OR, 0.9; $95 \%$ CI, $0.64-1.14 ; P=.27)$ or stroke (OR, $0.8 ; 95 \% \mathrm{CI}, 0.49$ $1.20 ; P=.25)$.

LENGTH OF STAY. Independent predictors of prolonged length of stay included age (OR, 1.05; 95\% CI, 1.04-1.05; $P<.01$ ), female sex (OR, 1.6; 95\% CI, 1.39-1.83; $P<$ $.01)$, BMI (OR, 1.02; 95\% CI, 11.01-1.03; $P<.01$ ), diabetes (OR, 1.3; 95\% CI, 11.11-1.47; $P<.01)$, chronic renal insufficiency (OR, 2.8; 95\% CI, 2.09-3.75; $P<.01$ ), hypertension (OR, 1.2; 95\% CI, 1.04-1.41; $P=.02)$, history of acute myocardial infarction (OR, 1.2; 95\% CI, 1.07-1.40; $P$ $<.01$ ), congestive heart failure (OR, 1.6; 95\% CI, 1.30$1.85 ; P<.01)$, reoperative CABG (OR, $1.4 ; 95 \% \mathrm{CI}$, $1.11-1.80 ; P=.01$ ), history of cerebrovascular accident (OR, 1.4; 95\% CI, 1.02-1.80; $P=.04$ ), preoperative IABP placement (OR, 2.0; 95\% CI, 1.59-2.41; $P<.01$ ), chronic obstructive pulmonary disease (OR, 2.0; $95 \% \mathrm{CI}$, 1.29-3.09; $P<.01$ ), peripheral vascular disease (OR, 1.4; 95\% CI, 1.19-1.70; $P<.01)$, and ejection fraction of less than 35\% (OR, 1.4; 95\% CI, 1.21-1.68; $P<.01$ ). There was no evidence of lack of fit, and there was no indication of multicollinearity among the independent variables.

IABP PLACEMENT. Independent predictors of IABP placement included hypertension (OR, 1.6; 95\% CI, 1.02$1.40 ; P=.04)$, history of acute myocardial infarction (OR, 1.8; 95\% CI, 1.21-2.75; $P<.01$ ), reoperative CABG (OR, $2.3 ; 95 \% \mathrm{CI}, 1.27-4.21 ; P=.01)$, and ejection fraction less than 35\% (OR, 1.8; 95\% CI, 1.22-2.60; $P<.01$ ). Off-pump CABG was associated with a lower rate of IABP placement (OR, 0.6; 95\% CI, 0.38-0.82; $P<.01$ ).

POSTOPERATIVE RENAL FAILURE. Independent predictors of postoperative renal failure included age $(\mathrm{OR}, 1.04 ; 95 \% \mathrm{CI}$, 1.03-1.06; $P<.01$ ), diabetes (OR, 1.5; 95\% CI, 1.18-2.02; $P<.01)$, preoperative chronic renal insufficiency (OR, 4.3; 95\% CI, 2.93-6.44; $P<.01$ ), and hypertension (OR, 1.8; 95\% CI, 1.22-2.54; $P<.01$ ). Off-pump CABG emerged as a predictor of lower rate of postoperative renal failure (OR, $0.5 ; 95 \%$ CI, 0.41-0.72; $P<.01)$.

HEMORRHAGE-RELATED RE-EXPLORATION. Independent predictors of hemorrhage-related re-exploration included BMI (OR, 0.96; 95\% CI, 0.93-0.99; $P<.01$ ). Off-pump CABG was associated with a lower rate of hemorrhage-related re-exploration (OR, 0.7; 95\% CI, 0.53-0.97; $P=.03$ ).

\section{Matched Sample}

The matched sample included 4026 patients (2013 in each group). There was no evidence of either multicollinearity or lack of fit in the logistic regression models predicting choice of surgical approach from preoperative risk factors. Propensity scores were computed for each patient by using a logistic regression model and matched on age, sex, hospital, hypertension, number of grafts, ejection fraction, reoperative CABG, diabetes, BMI, chronic renal failure, left main stenosis, history of acute myocardial infarction, urgent versus emergent case priority, history of cerebrovascular accident, preoperative IABP placement, preoperative hemodialysis, history of peripheral vascular disease, and carotid artery stenosis.

Operative mortality. Matches on the basis of propensity score were found for $73 \%$ of the patients undergoing offpump CABG. These patients had comparable operative mortality (OR, $0.8 ; 95 \% \mathrm{CI}, 0.57-1.15 ; P=.24)$ with the 
on-pump CABG group after controlling for preoperative risk factors through matching.

Stroke. Matches on the basis of propensity score were found for $68 \%$ of the patients undergoing off-pump CABG. Similarly, these patients had comparable risk of stroke (OR, 0.6 ; 95\% CI, 0.33-1.08; $P=.09$ ) with patients undergoing on-pump $\mathrm{CABG}$ after controlling for preoperative risk factors through propensity score matching.

Length of stay. Thirty-nine patients had to be excluded because of missing data on length of stay. Seventy-two percent of the off-pump cases were matched. Off-pump CABG was associated with an abbreviated length of stay compared with on-pump CABG after matching by propensity score (OR, $0.5 ; 95 \% \mathrm{CI}, 0.47-0.64 ; P<.01)$.

IABP Placement. Seventy-three percent of patients were matched. Off-pump CABG was associated with a lower rate of IABP placement compared with that seen in on-pump CABG after matching by propensity score (OR, $0.5 ; 95 \%$ CI, 0.3-0.71; $P<.01)$.

Postoperative renal failure. Seventy-three percent of patients were matched. Off-pump CABG was associated with a lower rate of renal failure compared with on-pump CABG after matching by propensity score (OR, 0.5; $95 \%$ CI, 0.37-0.72; $P<.01$ ).

Hemorrhage-related re-exploration. Off-pump CABG was associated with a lower rate of hemorrhage-related re-exploration compared with on-pump CABG after matching by propensity score (OR, $0.70 ; 95 \% \mathrm{CI}, 0.5-1.0 ; P=.5)$.

\section{Discussion}

\section{Higher Risk of Mortality Was Associated With Urgent} and Emergent CABG Compared With Elective CABG Although the short- and long-term outcomes of elective CABG have been improved, the morbidity and mortality rates of nonelective coronary revascularization remain high. ${ }^{14}$ Yamagishi and coworkers ${ }^{15}$ reported an in-hospital mortality rate of CABG after acute myocardial infarction as high as $38.9 \%$. Tomasco and associates ${ }^{16}$ conducted a retrospective study to identify the risk factors for hospital mortality in patients who underwent urgent and emergent CABG for acute myocardial infarction. They divided their patients into 3 groups: urgent group, defined as when surgical revascularization could be delayed for 24 to 36 hours because of adequate control of ischemia; emergent A group, defined as patients who required revascularization promptly after transient regression of ischemia by medical intervention; and emergent B group, defined as those who required prompt revascularization because the ischemia was completely refractory to medical intervention. The mortalities in the urgent, emergent $\mathrm{A}$, and emergent $\mathrm{B}$ groups were $7.4 \%$, $13.4 \%$, and $31.4 \%$, respectively, $(P<.001)$. The perioperative infarction rates were $11 \%, 11.8 \%$, and $63.3 \%$, respectively $(P<.001)$; the neurologic deficit rates were $2.8 \%, 3.4 \%$, and
$10.4 \%$, respectively $(P<.04)$; and the need for IABP was $1.9 \%, 4 \%$, and $16.9 \%$, respectively $(P<.001)$ when comparing the urgent, emergent $\mathrm{A}$, and emergent $\mathrm{B}$ groups.

\section{Off-pump CABG in Nonelective Coronary \\ Revascularization}

Off-pump CABG might be a safe alternative for coronary revascularization in patients who undergo nonelective CABG. Compared with on-pump CABG, off-pump CABG has been associated with a reduced rate of atrial fibrillation, ${ }^{17}$ lower stroke rates, ${ }^{18}$ and improved perioperative outcomes. ${ }^{19}$ The decreased organ dysfunction obtained with off-pump CABG has been largely ascribed to avoidance of the systemic inflammatory response elicited by the cardiopulmonary bypass circuit. Moreover, as previously reported, the incidence of atrial fibrillation after off-pump CABG is lower than after the conventional approach, mostly related to the period of myocardial ischemia, the required atrial cannulation, and the adverse effects of cardioplegia. ${ }^{20}$ Inadequate atrial protection has been demonstrated to be a trigger responsible for the development of atrial fibrillation in vulnerable patients ${ }^{21}$ and therefore of thromboembolic events after on-pump CABG. Off-pump CABG might benefit patients who undergo $C A B G$ after acute myocardial infarction on the basis of the fact that off-pump CABG seems to cause more regional than global ischemia compared with on-pump CABG. $^{7,22}$ Nakano and associates ${ }^{23}$ compared the outcomes of emergent and urgent off-pump CABG with those of elective off-pump CABG. Their results showed that there was no difference in graft patency between the emergenturgent group and the elective group (96.6\% vs 97.2\%). However, there was a significant difference in the mean number of grafts in the emergent group compared with that seen in the elective group $(1.8 \pm 0.2$ vs $2.7 \pm 0.2, P<.05)$.

\section{Outcomes of Nonelective Off-pump CABG Compared With Those of On-pump CABG}

In our study we used propensity score matching to adjust for the imbalances between the 2 groups. Nonelective off-pump CABG was associated with comparable operative mortality and stroke rates with conventional on-pump CABG, echoing previous studies. ${ }^{10,11}$ Previously, we demonstrated a decreased incidence of operative mortality and stroke after off-pump CABG compared with that after on-pump CABG in a risk-adjusted population. ${ }^{6,18}$ Failure to achieve statistical significance might be attributed to the relatively smaller number of patients analyzed in this report. In the present study nonelective off-pump CABG was associated with an abbreviated length of stay compared with that seen with on-pump CABG, as previously documented. ${ }^{11}$ In our study off-pump CABG emerged as an independent predictor of lower need for IABP placement and lower rate of postoperative renal failure and hemorrhage-related re-exploration compared with conventional on-pump CABG, echoing pre- 
vious reports. ${ }^{11}$ In a study by Karthik and associates, ${ }^{11}$ off-pump CABG was associated with lower rates of stroke $(0.7 \%$ vs $2.4 \%, P<.047)$, acute renal failure $(3.8 \%$ vs $7.8 \%, P<.015)$, prolonged ventilation $(5.3 \%$ vs $10.0 \%$, $P<.011$ ), need for postoperative intra-aortic balloon counterpulsation $(2.9 \%$ vs $7.8 \%, P<.002)$, and abbreviated hospital length of stay compared with on-pump CABG.

\section{Study Limitations}

Despite the fact that this is among the larger analyses to date comparing the outcomes of nonelective coronary revascularization with versus without cardiopulmonary bypass, all the limitations of a retrospective nonrandomized study apply. Multivariate analysis might not adequately account for the inherent selection bias in nonrandomized data. The retrospective nature of the study cannot account for the unknown variables affecting the outcomes that are not correlated strongly with measured variables. Propensity score adjustment is not a substitute for a properly designed randomized controlled trial; however, retrospective comparisons with propensity score adjustment are more versatile and might be more widely acceptable than randomized control trials. ${ }^{24,25}$ Randomized trials of impractical sizes are sometimes required to prove whether statistically significant differences really exist between these 2 techniques of myocardial revascularization.

Other limitations of the study include important variables not accounted for in our analysis, such as the size of the coronary vessels, which is important in selecting the type of operation, and the operating surgeon's selection bias to perform the operation on or off pump. Long-term efficacy and durability of nonelective minimally invasive versus conventional coronary artery surgery also remains to be answered.

In conclusion, nonelective off-pump CABG is associated with comparable operative mortality and stroke rates compared with those of conventional on-pump CABG. Moreover, off-pump CABG might reduce resource use because it is associated with an abbreviated length of stay compared with on-pump CABG and might decrease the need for IABP placement and lower the rates of postoperative renal failure and hemorrhage-related re-exploration.

\section{References}

1. Rose EA. Off-pump coronary-artery bypass surgery. $N$ Engl J Med. 2003;348:379-80.

2. Yusuf S, Zucker D, Peduzzi P, et al. Effect of coronary artery bypass graft surgery on survival: overview of 10 -year results from randomized trials by the Coronary Artery Bypass Graft Surgery Trialists Collaboration. Lancet. 1994;344:563-70.

3. Cremer J, Martine M, Redl H, et al. Systemic inflammatory response syndrome after cardiac operations. Ann Thorac Surg. 1996;61:1714-20.
4. Lui B, Belboul A, Larsson S, Roberts D. Factors influencing hemostasis and blood transfusion in cardiac surgery. Perfusion. 1996;11: 131-43.

5. Puskas JD, Williams WH, Duke PG, et al. Off-pump CABG provides complete revascularization with reduced myocardial injury, transfusion requirements, and length of stay: a prospective randomized comparison of two hundred unselected patients undergoing off-pump versus conventional coronary artery bypass grafting. J Thorac Cardiovasc Surg. 2003;125:797-808.

6. Magee MJ, Jablonski KA, Stamou SC, et al. Elimination of cardiopulmonary bypass improves early survival for multivessel coronary artery bypass patients. Ann Thorac Surg. 2002;73:1196-202.

7. Calafiore A, Di Mauro M, Contini M, et al. Myocardial revascularization with and without cardiopulmonary bypass in multivessel disease: impact of the strategy on early outcome. Ann Thorac Surg. 2001;72:1926-32.

8. Plomondon ME, Cleveland JC, Ludwig ST, et al. Off-pump coronary artery bypass is associated with improved risk-adjusted outcomes. Ann Thorac Surg. 2001;72:114-9.

9. Sahre H, Kappert U, Eller M, et al. An alternative approach for the treatment of acute myocardial infarction-OPCAB. Heart Surg Forum. 2003;6(suppl 1):S42.

10. Hirose H, Amano A, Takahashi A, et al. Urgent off-pump coronary artery bypass grafting. Jpn J Thorac Cardiovasc Surg. 2002;50:330-7.

11. Karthik S, Musleh G, Grayson AD, et al. Effect of a avoiding cardiopulmonary bypass in non-elective coronary artery bypass surgery: a propensity score analysis. Eur J Cardiothorac Surg. 2003;24:66-71.

12. Grunkemeier GL, Payne N, Jin R, Handy JR Jr. Propensity score analysis of stroke after off-pump coronary artery bypass grafting. Ann Thorac Surg. 2002;74:301-5.

13. Allison P. Logistic regression using the SAS system, theory and application. Indianapolis, IN, SAS Institute; 1999. p. 197-206.

14. Nakano H, Daimon M, Hayashi K, et al. Evaluation of value of CABG for emergency or subemergency case. Kyobu Geka. 2001;54:298-304.

15. Yamagishi I, Sakurada T, Abe T. Emergency CABG after acute myocardial infarction. What influences early postoperative mortality? Ann Thorac Cardiovasc Surg. 1998;4:28-33.

16. Tomasco B, Cappiello A, Fiorilli R, et al. Surgical revascularization for acute coronary in sufficiency: analysis of risk factors for hospital mortality. Ann Thorac Surg. 1997;64:678-83.

17. Allen KB, Matheny RG, Robison RJ, Heimansohn DA, Shaar CJ. Minimally invasive versus conventional reoperative coronary artery bypass. Ann Thorac Surg. 1997;64:616-22.

18. Stamou SC, Jablonski KA, Pfister AJ, et al. Stroke after conventional versus minimally invasive coronary artery bypass. Ann Thorac Surg. 2002;74:394-9.

19. Mack MJ, Pfister A, Bachand D, et al. Comparison of coronary bypass surgery with and without cardiopulmonary bypass in patients with multivessel disease. J Thorac Cardiovasc Surg. 2004;127:167-73.

20. BhaskerRao B, VanHimbergen D, Edmonds HL Jr, et al. Evidence for improved cerebral function after minimally invasive bypass surgery. J Card Surg. 1998;13:27-31.

21. Taylor RL, Borger MA, Weisel RD, Fedorko L, Feindel CM. Cerebral microemboli during cardiopulmonary bypass: increased emboli during perfusionist interventions. Ann Thorac Surg. 1999;68:89-93.

22. Vlassov G, Deyneka C, Travine N, et al. Acute myocardial infarction; OPCAB is an alternative approach for treatment. Heart Surg Forum. 2001;4:147-51.

23. Nakano H, Daimon M, Hayashi K, et al. Evaluation of value of CABG for emergency or subemergency case. Kyobu Geka. 2001;54:298-304.

24. Grunkemeier GL, Payne N, Jin R, Handy JR Jr. Propensity score analysis of stroke after off-pump coronary artery bypass grafting. Ann Thorac Surg. 2002;74:301-5.

25. Blackstone E. Comparing apples and oranges. J Thorac Cardiovasc Surg. 2002;123:8-15. 